\title{
British Allergy Society
}

The Summer Meeting of the British Allergy Society was held at the Medical School, Birmingham University on Saturday 13th June, 1964. The speakers were Dr. R. M. Norris (Birmingham) on 'The effects of breathing inert dust on the distribution of ventilation and perfusion of the lungs', Dr. C. N. D. Cruickshank (Birmingham) on 'Allergy to fungi', Dr. K. R. Haye (Birmingham) on 'Basophile degranulation (Shelly technique) in atopic subjects', Dr. K. M. Citron (London) on 'Lung disease associated with eosinophilia', Dr. J. F. Soothill (Birmingham) on 'The presence in the serum of patients with glomerulo-nephritis of a qualitatively abnormal component of Complement (jSia)' and Dr. H. Morrow Brown on 'The importance of yeasts as allergens'.

Honorary Secretary: J. M. Smith, British Allergy Society, 21 Hagley Road West, Harborne, Birmingham 17.

The Effects of Breathing Inert Dust on the Distribution of Ventilation and Perfusion of the Lungs

R. M. Norris and J. M. Berhof

The Queen Elizabeth Hospital, Birmingham 15

Allergy to Fungi

C. N. D. Cruickshank M. R. C. Unit for Research on the Experimental Pathology of the Skin, The Medical School, University of Birmingham.

From deep cultures of Trichophyton mentographytes and Trichophyton rubrum, galacto-mannan peptides have been isolated and shown to be allergenic. These have the general structure of Dgalactose and D-mannose in the proportion of about 1 to 8 , and the protein is present to approximately $10 \%$. There is very close structural relationship between galactomannans from $\mathrm{T}$. mentagrophytes and from $T$. rubrum. In addition, they appear to be virtually completely crossreactive in guinea pigs and in humans. Clinical studies have been made with purified trichophytin and compared with the results obtained from commercial trichophytin. It appears that the incidence of immediate reactions obtained with the purified preparation is considerably reduced, while the incidence of delayed reactions is approximately the same.

Basophile Degranulation (Shelly Technique) in Atopic Subjects

K. R. Haye

M. R. C. Unit for Research of the Experimental Pathology of the Skin,

The Medical School, University of Birmingham.

The Shelley basophile degranulation technique and modifications of it have been examined in normal subjects, atopies and persons suffering from various hypersensitivities. The result of the direct test appear in atopic subjects to bear a close relationship with the clinical history and skin test results. The indirect test has proven less reliable, in that a higher proportion of the sera were toxic to rabbit basophils. In those tests in which serum did not produce this effect the correlation with the clinical history and the results of the direct test was unsatisfactory in that only about one half corresponded.

Proceedings - Gesellschaftsberichte - Sociétés 
Lung Disease Associated with Eosinophilia

K. M. Citron The Brompton Hospital, London.

The term pulmonary eosinophilia is applied to those conditions in which there occurs ill-defined pulmonary shadowing on the radiograph accompanied by blood eosinophilia. Cases of pulmonary eosinophilia vary widely in their clinical features and include mild transient disease similar to that described by Loeffler, recurrent disease, or progressive fatal disease with involvement of many organs of the body.

The following classification is suggested:

Parasitic infections: Ascaris, Schistosomes, filaria, lung fluke, hookworms, Strongy-loides, Echinococcus, Trichina, Entamoeba histolytica, whipworms, toxicara canis, liver fluke, beef tapeworm.

Tropical Pulmonary Eosinophilia: ? Filaria.

Fungal Injections: Aspergillus fumigatus, Coccidiomyiosis.

Inhalants: Pollen, spores (extrinsic asthma).

Medicaments: P. A. S. penicillin, sulphonamide, nitrofurantoin, sera.

Intrinsic Asthma.

Polyarteritis nodosa.

Unidentified Cause.

Between 1959 and 1963 forty-eight patients with pulmonary eosinophilia were investigated at Brompton Hospital, London. The causes found were allergic pulmonary aspergillosis in thirtyseven, tropical pulmonary eosinophilia in four, polyarteritis nodosa in two and the cause was obscure in five.

The Detection of the Altered Form of the Complement Component C'3A (Bic-BiA) 1/8 the Serum of Patients with Various Forms of Glomerulo-nephritis

\section{J. F. SOOTHILL}

Department of Experimental Pathology, The Medical School, University of Birmingham. It has been assumed that the low level of serum complement activity in the serum of patients with acute glomerulo-nephritis, is due to its being used up in an antigen-antibody reaction, though the evidence for this assumption is circumstantial. The observation by Muller-Eberhard and others that exposure of fresh human serum in vitro to an antigen-antibody reaction led to an alteration of the electrophoretic mobility of the complement component called $\mathrm{C}^{1 / 8 a}$ or $\beta \backslash \mathrm{c}$ (to form the so-called BiK), provides a means of obtaining direct evidence for this assumption. Fresh plasma from patients with acute post-streptoccocal glomerulo-nephritis with low serum complement was found to contain the altered component, whereas controls were consistently negative using the same technique. Since this work was started, Lachmann has reported a similar phenomenon in Disseminated Lupus Erythematosus.

Other patients with acute nephritis syndrome with normal serum complement activity were also found to contain the abnormal constituent as was the serum of many patients with persistent proliferative glomerulo-nephritis. It was also found in the serum of patients with membranous glomerulo-nephritis, though at a lower concentration, providing the first direct evidence of an immunological basis for this disease process. This constituent has not been found in patients with nephrotic syndrome with minimal renal histological abnormality, or in nephrotic syndrome due to renal vein thrombosis.

124 Proceedings - Gesellschaftsberichte - Sociétés

Allergy to Ingested and Airborne Yeasts as a Common Cause of Allergic Disorders

H. Morrow Brown Derby Chest Clinic, 93 Green Lane, Derby. 
The observations of aerobiological factors in the spread of plant disease have clearly indicated that the air, particularly in the summer, contains large quantities of potential allergens. Close relationships of weather changes with symptoms, and ingestion of yeast tablets or beer followed by symptoms, suggested that yeasts might be important, but skin tests were valueless. Nasal provocation tests were found to produce many positive results by provoking symptoms, and results of this type of tests in nearly 600 patients were presented, showing that half of them were yeast sensitive. Data referring to de-sensitization by Depot emulsions of these allergens and subsequent demonstration of a negative nasal provocation test was presented.

An out-sized Bourdillon Slit Sampler to take automatic volumetric half hourly samples which had been devised by the author and used for making aerobiol - > gical observations on culturable allergens, was described and the intimate link-up between patient's symptoms and aerobiological changes was demonstrated.

I. It was pointed out that yeasts which have so far been used in provocation tests are not necessarily the correct ones and that a great deal of research requires to be done on air-borne yeasts. For example

certain patients had severe symptoms when in heavy rain but all attempts to identify these allergens had been unsuccessful. 